\title{
A DESIGN OF EXPERIMENTS APPROACH TO MILITARY DEPLOYMENT PLANNING PROBLEM
}

\author{
Uğur Z. Yıldırım \\ İhsan Sabuncuoğlu \\ Barbaros Tansel
Department of Industrial Engineering
Bilkent University
Bilkent, Ankara 06800, TURKEY

\author{
Ahmet Balcioğlu \\ Turkish Army Headquarters \\ Turkish Army \\ Yücetepe, Ankara,06100, TURKEY
}

\begin{abstract}
We develop a logistics and transportation simulation that can be used to provide insights into potential outcomes of proposed military deployment plans. More specifically, we model the large-scale real-world military Deployment Planning Problem. It involves planning the movement of military units from their home bases to their final destinations using different transportation assets on a multimodal transportation network. We use an intelligent design of experiments approach to evaluate logistics factors with the greatest impact on the overall achievement of a typical real-world military deployment plan.
\end{abstract}

\section{INTRODUCTION}

Regional and asymmetric threats and the increase in worldwide terrorist activity have made logistics and mobility increasingly important in our rapidly changing world. This paper deals with logistics and transportation simulations that are used to provide insight into the potential outcomes of proposed logistical courses of actions prior to and after committing members of the military into harm's way. More specifically, we model the Deployment Planning Problem (DPP), defined and thoroughly described first by (Akgün and Tansel 2007). DPP involves movement and positioning of many military units from their home bases to their designated destinations to carry out a mission. This movement mostly occurs on a multimodal (land, rail, sea, air, and inland waterways) transportation network using different transportation assets. During peace time, plans are made to deploy the required number of troops and equipment to potential threat or disaster areas. During a time of crisis or natural disaster, it will be necessary to use these plans as they are, to modify them as necessary or to create new deployment plans in a short time. For these reasons, we have developed a simulation model of military deployment with accurate transportation network infrastructure data and a medium-resolution allowing planners to develop and analyze plans in a relatively short time (Yıldırım, Sabuncuoğlu, and Tansel 2007) .
Even a small-scale military deployment scenario can have a large number of input variables (factors) that may impact the outcome of the plan. In classical design of experiments (DOE), one can explore only a handful of cases. With the use of computers and in a simulation setting, the user can vary a large number of input variables. Yet, even with today's computing power, complete enumerations of all possible scenarios is an exhaustive task. To intelligently sample the state space of possible alternatives in a simulation setting, we use an approach developed for large-scale simulation experiments with many factors (Kleijnen et al. 2005), (Cioppa and Lucas 2007).

In Section 2, the DPP is explained in detail. The simulation model of deployment problem is briefly presented in Section 3. In Section 4, relevant information on DOE approach used is explained, and details of our case study are presented. Our results are presented in Section 5. Final remarks are made in Section 6.

\section{PROBLEM AND SYSTEM DEFINITION}

The DPP involves the simultaneous and coordinated relocation of many military units from their home bases to their designated destinations according to a given military mission. This mission may be a preplanned and rehearsed one or may be due to a contingency that arose because of an escalating military confrontation or a natural disaster. In the latter case, timely deployment of military units to their destinations in the crisis area is of utmost importance. In the former case, the more important concern is cost. Usually, a least cost deployment plan is preferred during peace time when time is not of essence.

If there is ample time to plan for a particular deployment, this is called deliberate planning. When the time available for planning and execution of an actual deployment is short, this is called crisis action planning or timesensitive planning. During crisis action planning, quick response and flexibility to adapt to rapidly changing situations are crucial. Each deployment plan, deliberate or not, has as a minimum a list of cargo and pax (troops) to be transported by type and quantity, and movement data by 
mode of transportation, earliest times of departures from home bases, and earliest and latest times of arrivals at destinations.

The movement of military units from their home bases to their final destinations are conducted in groups. A unit is mostly divided into three components (advance party, pax party, and cargo party) during deployment. Ground movement is conducted in convoys. The speed and composition of convoys are decided by operational and tactical objectives and constraints. Coordinated movements of components of several units are dictated mainly by availability and capacity of lift assets, capacities of transfer points, weather, operational requirements, and intelligence on enemy's probable courses of action.

Large-scale deployments over long distances may require the outsourcing of heavy lift assets of civilian companies or other nations'. Most of the time, a unit's own (organic) transportation assets will suffice to conduct a deployment. Deployments over long distances (usually outside a country's borders) are classified as strategic deployments. Strategic deployments are mostly conducted using sea and air lift assets. Once a strategically deploying unit reaches its destination country, other available modes of transportation may be utilized. Deployments inside a country's borders are referred to as intra-theater deployments. This type of deployment may utilize any of available (land, sea, air, and rail) modes of transportation.

Transportation mode changes may be required at transfer points during both strategic and intra-theater deployments. Furthermore, successive mode changes may be necessary at different transfer points during a deployment. However, the fewer the mode changes are at transfer points, the easier is the deployment. Main transfer points are harbors, train stations and airports. At these locations, the pax (troops) and cargo (weapon systems, material, equipment, and supplies) a unit has, collectively referred to here as items, are transferred from one set of transportation assets to another set that operate on a different network. This location is also called a Port of Embarkation (POE). The next mode change location, where the items are offloaded and loaded onto another set of transportation assets is called a Port of Debarkation (POD). These may be sea, rail and air POEs or PODs (namely, SPOE/SPOD, RPOE/RPOD etc.).

At transfer points, units usually queue up before being loaded on vessels. This location is called a staging area where units wait and prepare for shipment. A staging area can be regarded as a service point, i.e. one with a certain capacity of material handling equipment and load/unload docks. When there is not enough capacity at a staging area to hold large number of deploying units, a marshaling area is operated. A marshalling area can be regarded as a waiting/parking place. It helps provide an uninterrupted flow of items through their transfer points (Akgün and Tansel 2007).
Real-world deployments are characterized by unpredictable stochastic events (breakdowns, accidents, delays etc.), load/unload/idle times at home bases, destinations, and transfer points. Thus, a stochastic simulation is suitable to determine potential outcomes of deployment plans.

\section{THE SIMULATION MODEL}

We created our discrete-event simulation of DPP by utilizing Schruben's (1983) Event Graph (EG) methodology. In an EG, events are vertices (nodes) on a directed graph and they represent state changes of the system. Directed edges (arcs) between vertices indicate how occurrence of one event triggers another event. Solid arcs can be referred to as scheduling arcs, whereas dashed arcs are called canceling arcs. A tilde on an arc represents the conditional scheduling of the event at the head of the arc whenever the stated Boolean condition is true. EGs are not flow charts. They represent the conceptual and logical models of our simulation. More detailed information on EGs are available in Sargent (1988) and Buss (2001).

The modular structure of our simulation is constructed via the Listener Event Graph Object (LEGO) framework (Buss and Sanchez 2002). The listener pattern of object oriented programming allows LEGOs to work. EGs and LEGOs can be programmed using Simkit, a Java Application Programming Interface (API) developed at the Naval Postgraduate School and freely available via <http://diana.nps.edu/Simkit/>.

We have integrated a geographical information system (GIS), named GeoKIT, into our simulation. Benefits of combining simulation and GIS include usage of real and current transportation network data, an accurate animation, and extended analysis possibilities in route selection. GeoKIT is a Java API, which enables easier integration with Simkit. Furthermore, selection of GeoKIT as the GIS system of choice is due to its superb capabilities and comprehensive set of components to manipulate and use geospatial information. More information on GeoKIT can be found at $<$ http: //geokit.bilgigis.com>.

The details of our simulation model, developed using the tools briefly described above, are in (Yıldırım, Sabuncuoğlu, and Tansel 2007).

\subsection{Verification and Validation}

Verification and validation were conducted by using appropriate methods explained in Sargent (2001). To mention a few more specifically, for face validity, we have discussed inputs and outputs of the model and its EGs with potential users of the model. We used assertion checking to verify that the model functioned within its acceptable domain. Incrementally, bottom-up testing was performed, where each individual submodel was tested and integrated. Fault (failure) insertion testing was used to test whether 
the model responded by producing an invalid behavior given the faulty component. During special input testing, we used an arbitrary mixture of minimum and maximum values, and invalid data for the input variables, and tested for potential peculiar situations at the boundary values. In addition, we have tested the validity and behavior of the model under extreme workload and congestion at the load/unload docks and transfer points such as SPOEs, SPODs etc. Animation also helped in discovering errors during model development. Furthermore, results of the deployment optimization model developed by Akgün and Tansel (2007) were used for verification purposes. Simulation results were compared to the historical military deployment data.

\section{CASE STUDY AND DOE}

Here we present a typical real-world military deployment case study and the DOE approach used in its analysis.

\subsection{Case Study}

A deployment scenario for deploying four battalions (three mechanized and one armored) during peace-time from the Iraqi border in the southeast to northwestern Turkey is analyzed. The scenario uses land, sea, and rail transportation networks and assets. These four units deploy from three different home bases to three unique destinations. Units $\mathrm{C}$ and D are co-located, and Units B and C deploy to the same destination location. The data related to the deployment of each unit and its components are listed in Table 1. As shown in the line allocated to Unit A in Table 1, Unit A deploys in three components; advance, pax and cargo parties. The advance party for Unit A has 40 pax, one 2.5 -ton truck, one generator, three Type 1 boxes, and three Type 2 boxes. The pax party for Unit A has 450 personnel. The cargo party for Unit A will deploy by sea. It has 10 pax, 45 armored personnel carriers (APCs), 14 tanks, nine 2.5ton trucks, ten 5-ton trucks, seven generators, 37 Type 1 boxes, and 37 Type 2 boxes. Unless otherwise indicated in Table 1, each component deploys by land. The total number of deployed personnel and equipment for Unit A are shown in the line named total. Units B, C, and D deploy in two components each. Their cargo parties deploy by rail as indicated in Table 1. Table 2 lists the dimensions, weight and volume information of deployed equipment used in our simulation. For example, the first line in Table 2 indicates that an APC used in this deployment weighs $11500 \mathrm{~kg} . \mathrm{s}$ (11.5 tons), has a volume of $43 \mathrm{~m}^{3}$. Its width, length, and height are 280, 535, and 287 centimeters, respectively. The minimum and maximum time requirements for deployed units to be at their designated destination locations (not shown) and the initial delay times for each deployment component, that are used to ensure timely and coordinated arrivals at destinations for each deploying unit (not shown), are also taken into ac-

Table 1: Units and Their Equipment

\begin{tabular}{|c|c|c|c|c|c|c|c|c|c|}
\hline $\begin{array}{l}\text { Unit } \\
\text { Name }\end{array}$ & $\begin{array}{l}\text { Deployment } \\
\text { Component }\end{array}$ & $\begin{array}{c}\text { Pax } \\
\text { Number }\end{array}$ & APC & Tank & $\begin{array}{c}\text { 2.5-Ton } \\
\text { Truck }\end{array}$ & $\begin{array}{l}\text { 5-Ton } \\
\text { Truck }\end{array}$ & Generator & $\begin{array}{c}\text { Box } \\
\text { (Type 1) }\end{array}$ & $\begin{array}{c}\text { Box } \\
\text { (Type 2) }\end{array}$ \\
\hline \multirow{4}{*}{ Unit A } & Total & 500 & 45 & 14 & 10 & 10 & 8 & 40 & 40 \\
\hline & Advance Party & 40 & & & 1 & & 1 & 3 & 3 \\
\hline & Pax Party & 450 & & & & & & & \\
\hline & $\begin{array}{l}\text { Cargo Party by } \\
\text { Sea }\end{array}$ & 10 & 45 & 14 & 9 & 10 & 7 & 37 & 37 \\
\hline \multirow{3}{*}{ Unit B } & Total & 500 & 45 & 14 & 10 & 10 & 8 & 40 & 40 \\
\hline & Pax Party & 450 & & & & & & & \\
\hline & $\begin{array}{l}\text { Cargo Party by } \\
\text { Rail }\end{array}$ & 50 & 45 & 14 & 10 & 10 & 8 & 40 & 40 \\
\hline \multirow{3}{*}{ Unit C } & Total & 500 & 45 & 14 & 10 & 10 & 8 & 40 & 40 \\
\hline & Pax Party & 450 & & & & & & & \\
\hline & $\begin{array}{l}\text { Cargo Party by } \\
\text { Rail }\end{array}$ & 50 & 45 & 14 & 10 & 10 & 8 & 40 & 40 \\
\hline \multirow{3}{*}{ Unit D } & Total & 500 & 14 & 45 & 10 & 10 & 8 & 40 & 40 \\
\hline & Pax Party & 450 & & & & & & & \\
\hline & $\begin{array}{l}\text { Cargo Party by } \\
\text { Rail }\end{array}$ & 50 & 14 & 45 & 10 & 10 & 8 & 40 & 40 \\
\hline
\end{tabular}


Table 2: Dimensions and Weights of Deployed Equipment

\begin{tabular}{|l|c|c|c|c|c|c|}
\hline Item Type & Weight (kg.) & $\begin{array}{c}\text { Width } \\
(\mathbf{c m} .)\end{array}$ & $\begin{array}{c}\text { Length } \\
(\mathbf{c m})\end{array}$ & $\begin{array}{c}\text { Height } \\
(\mathbf{c m} .)\end{array}$ & $\begin{array}{c}\text { Volume } \\
(\mathbf{m} 3)\end{array}$ & Explanation \\
\hline APC & 11500 & 280 & 535 & 287 & 43 & \\
\hline Tank & 65000 & 305 & 540 & 343 & 56.5 & \\
\hline 2.5 -Ton Truck & 5000 & 235 & 570 & 270 & 36 & Self-deployed \\
\hline 5 -Ton Truck & 5000 & 250 & 790 & 330 & 65 & Self- deployed \\
\hline Generator & 300 & 100 & 150 & 100 & 1.50 & \\
\hline Box (Type 1) & 50 & 50 & 150 & 100 & 0.75 & \\
\hline Box (Type 2) & 100 & 100 & 150 & 100 & 1.50 & \\
\hline
\end{tabular}

count in the simulation model. The simulation for this scenario is a terminating one with a termination time of 240 hours. The transportation assets allocated to this scenario are not listed in detail here. They include one large RoRo ship, four trains with enough and appropriate rail cars, trucks, and tank carriers (one tank carrier can carry a single tank or 2 APCs). The armored vehicles must be carried by rail or sea to distances over $300 \mathrm{~km}$. In this scenario, they deploy over $300 \mathrm{~km}$. Other restrictions used in the simulation, such as the storage capacities of SPOE, SPOD, RPOE, and RPOD, are not presented here. Our performance measure is the percentage of on-time arrivals (averaged across replications) for each unit to their designated destinations.

\subsection{Design of Experiments}

DOE deals with different fields from medicine to farming, and provides procedures for efficient conduct of statistical experiments. Computer simulation provides a vast area in which to expand DOE. Some of the possible designs are presented in the Design Toolkit (Kleijnen et al. 2005). Latin Hypercube (LH) designs are recommended for simulation experiments with minimal assumptions and many factors. Ye developed an algorithm for orthogonal $\mathrm{LH}$ designs (Ye 1998). This was later extended by Cioppa and Lucas, who gave up small amounts of orthogonality for better space-filling designs, and developed the Nearly Orthogonal Latin Hypercube (NOLH) designs (Cioppa and Lucas 2007). Orthogonal Latin Hypercube (OLH) and NOLH designs are special cases of LH designs. OLH designs have strict orthogonal properties, i.e., a matrix condition number of 1 , and a maximum pairwise correlation of zero between any two columns in the design matrix. NOLH designs relax the requirements on the orthogonal properties. NOLH designs choose the most space-filling design among design matrices that satisfy near orthogonal thresholds. In a good space-filling design, the design points are required to be scattered throughout the experimental region with minimal unsampled regions. The limits used by Cioppa and Lucas are a condition number of less than 1.13 and a maximum pairwise correlation between all columns of the design matrix in the interval $(-.03, .03)$ (Cioppa and Lucas 2007). The NOLH design matrix is a compromise between complete enumerations of all possible scenarios, which is an exhaustive task even with today's computing power, and an OLH design. NOLH designs can also handle discrete variables, as opposed to LH designs which can only handle continuous variables, at a cost of orthogonality and space-filling properties if the levels of discrete variables are a few. Generating these designs is a time-consuming process. But a catalogue of ready-to-use designs are available online. This paper utilizes a 29-factor and 257-run design by (Sanchez and Hernandez 2005). Table 3 provides the factors and their levels for experimentation with our deployment simulation. The levels of these factors are entered into the spreadsheet provided by Sanchez and Hernandez to create a nearlyorthogonal and space-filling 257-run design matrix. The factors are the convoy speed, the number of load and unload docks at transfer points (such as SPOE/RPOE etc.) and minor/medium/major breakdown probabilities for transportation assets used in the deployment. These factors were chosen according to expert opinion. As more units are added, more factors with varying levels will have to be considered.

\section{RESULTS}

Part of the design matrix (first 10 runs for the first 5 factors) created using the factors and their levels in Table 3 are presented in Table 4. For each of these input combinations (rows) of our 257-run design matrix (partly depicted in Table 4), we have written a script to modify the simulation's base-case XML scenario file. We made 15 replications (to achieve the desired accuracy of $90 \%$ relative pre- 
cision) of each of the newly-created 257 scenarios to reach a total of $257 \times 15=3855$ computer runs. Compare this to an experiment with 29 factors each with only 2 levels and 15 replications per run for a complete enumeration experiment $\left(2^{29} \times 15=8,053,063,680\right.$ computer runs!). We have written a VBA script to extract and calculate the percentage of ontime arrivals for each run (averaged across replications) from the simulation output files written onto EXCEL spreadsheets. For Unit A, Averaged on-time Arrivals at its destination (AoA) ranged between $76.8 \%$ and $100 \%$ with a average of $97.93 \%$ and a standard deviation of 0.0374 . That is, on average, 97.93 percent of all vehicles of Unit A arrived at their destination on-time according to the deployment plan. For Units B and C deploying to the same destination, AoA ranged between $96 \%$ and $100 \%$ with an average of $99.96 \%$ and a standard deviation of 0.0034 . The deployment plans for Units B and C seem to be robust across the input combinations simulated. For Unit D, AoA ranged between $93.04 \%$ and $98.8 \%$ with an average of $98.57 \%$ and a standard deviation of 0.0118 .

Table 3: Factors and Their Levels

\begin{tabular}{|c|l|l|l|}
\hline$\#$ & \multicolumn{1}{|c|}{ Factor Name } & Low & High \\
\hline 1 & Convoy speed & 30 & 70 \\
\hline 2 & Unit A Sea Group \# load docks & 3 & 37 \\
\hline 3 & Unit A Destination \# unload docks & 3 & 10 \\
\hline 4 & Unit B Home \# load docks & 3 & 37 \\
\hline 5 & Unit B RPOE \# load docks & 1 & 10 \\
\hline 6 & Unit B RPOD \# unload docks & 1 & 6 \\
\hline 7 & Unit B Destination \# unload docks & 3 & 37 \\
\hline 8 & Unit C Home \# load docks & 3 & 20 \\
\hline 9 & Unit C RPOE \# load docks & 1 & 10 \\
\hline 10 & Unit D Home \# load docks & 3 & 37 \\
\hline 11 & Unit D RPOE \# load docks & 1 & 6 \\
\hline 12 & Unit D RPOD \# unload docks & 1 & 6 \\
\hline 13 & Unit D Destination \# unload docks & 3 & 37 \\
\hline 14 & Truck Minor Breakdown Probability & 0 & 0.05 \\
\hline 15 & Truck Medium Breakdown Prob. & 0 & 0.07 \\
\hline 16 & Ship Minor Breakdown Probability & 0 & 0.1 \\
\hline 17 & Ship Medium Breakdown Prob. & 0 & 0.008 \\
\hline 18 & Train Minor Breakdown Probability & 0 & 0.06 \\
\hline 19 & Train Medium Breakdown Prob. & 0 & 0.02 \\
\hline 20 & Train Major Breakdown Probability & 0 & 0.001 \\
\hline 21 & Bus Minor Breakdown Probability & 0 & 0.05 \\
\hline 22 & Bus Medium Breakdown Probability & 0 & 0.025 \\
\hline 23 & Bus Major Breakdown Probability & 0 & 0.008 \\
\hline 24 & Tank Minor Breakdown Probability & 0 & 0.1 \\
\hline 25 & Tank Medium Breakdown Prob. & 0 & 0.04 \\
\hline 26 & Tank Major Breakdown Probability & 0 & 0.008 \\
\hline 27 & APC Minor Breakdown Probability & 0 & 0.15 \\
\hline 28 & APC Medium Breakdown Prob. & 0 & 0.025 \\
\hline 29 & APC Major Breakdown Probability & 0 & 0.009 \\
\hline
\end{tabular}

To identify which factors contribute more to our performance measure of AoA, we use a nonparametric approach, namely regression trees, to reveal the structure in the data in a more human-readable way. We append the AoA response variable for Units as $30^{\text {th }}$ column to the 257 run design matrix for 29 factors, and import this newly formed 30-column matrix into JPM (SAS 2005).

Table 4: 10 Runs for First 5 Factors of 257-Run Matrix

\begin{tabular}{|c|c|c|c|c|c|}
\hline low level & 30 & 3 & 3 & 3 & 1 \\
\hline high level & 70 & 37 & 10 & 37 & 10 \\
\hline decimals & 0 & 0 & 0 & 0 & 0 \\
\hline name & 1 & 2 & 3 & 4 & 5 \\
\hline 1 & 46 & 33 & 7 & 24 & 7 \\
\hline 2 & 35 & 17 & 9 & 36 & 7 \\
\hline 3 & 36 & 23 & 4 & 22 & 10 \\
\hline 4 & 46 & 8 & 6 & 25 & 8 \\
\hline 5 & 45 & 22 & 7 & 17 & 9 \\
\hline 6 & 48 & 15 & 10 & 8 & 9 \\
\hline 7 & 32 & 24 & 6 & 7 & 6 \\
\hline 8 & 45 & 4 & 6 & 17 & 9 \\
\hline 9 & 33 & 21 & 10 & 24 & 4 \\
\hline 10 & 49 & 6 & 9 & 27 & 1 \\
\hline
\end{tabular}

This is done separately for Unit A, Unit D, and Units B\&C. The data are split into two leaves in such a way that the variability in the response within each leaf decreases and the variability in the response between leaves increases (Kang, Doerr, and Sanchez 2006). The split is continued until the point of diminishing returns in the value of $R^{2}$ (a measure of the amount of variance in the data that is explained by the given model) is reached. Only after 26 splits, an $\mathrm{R}^{2}$ of 0.617 is reached for Unit A. For Units B and C, an $\mathrm{R}^{2}$ value of 0.317 is reached only after JMP makes 6 splits, and no further splits can be made. However, a careful examination of the split columns (factors) reveal that two factors have nothing to do with the deployment of Units B and C, and thus can be pruned to achieve an almost the same level of $\mathrm{R}^{2}$ of 0.314 . Figure 1 shows the final regression tree for Units B and C. For (column) factor 11 (the number of load docks at RPOE for Unit D), the AoA is $100 \%$ for 60 scenarios when number of load docks is greater than 2, as opposed to an AoA of $99.4 \%$ for 6 scenarios when the number of load docks is less than 2. Although not a huge difference, this is due to the fact that Units C and D are colocated and share the same resources (loading docks) even though the design matrix is for deployment of Units B and C. Major factors of influence seem to be minor breakdown of trucks and medium breakdown of Armored Personnel Carriers (APCs). As for Unit D, JMP makes 43 splits of the data to achieve an $R^{2}$ of 0.417 . A careful examination of splits and pruning of unnecessary and illogical leaves (i.e. factors not related to deployment of Unit D), 


\section{Yıldırım, Sabuncuoğlu, Tansel, and Balcıoğlu}

reveals that the same $R^{2}$ value can be achieved with only 11 splits of the data.

Regression metamodels (though harder to interpret) may help validate the regression tree results to determine which factors have the greatest influence on the response variable of interest. We also fit regression models. With 29 input variables, generating a model including interactions for a given performance measure can be tedious. Thus, we used mixed stepwise regression, where JMP software package alternates forward and backward stepwise regression until the remaining terms are significant, to select a subset of the input variables. In the backwards selection, terms are brought into the model and then the least significant terms are removed until all the remaining terms are significant. It is the opposite in the forward selection case where the most significant term is brought into the model. After stepwise regression is used to determine a model of interest, the model is fit to a linear regression using standard least squares. Once fit, three statistics, adjusted $\mathrm{R}^{2}$, the F test statistic, and the Student's t-test statistic, are examined to decide on the goodness and applicability of the model. In our case, linear models did not suffice and we had to fit nonlinear models with interaction terms (details not included due to page limitations). Other explanatory tools such as interaction profiles are not included in this paper for the same reason.

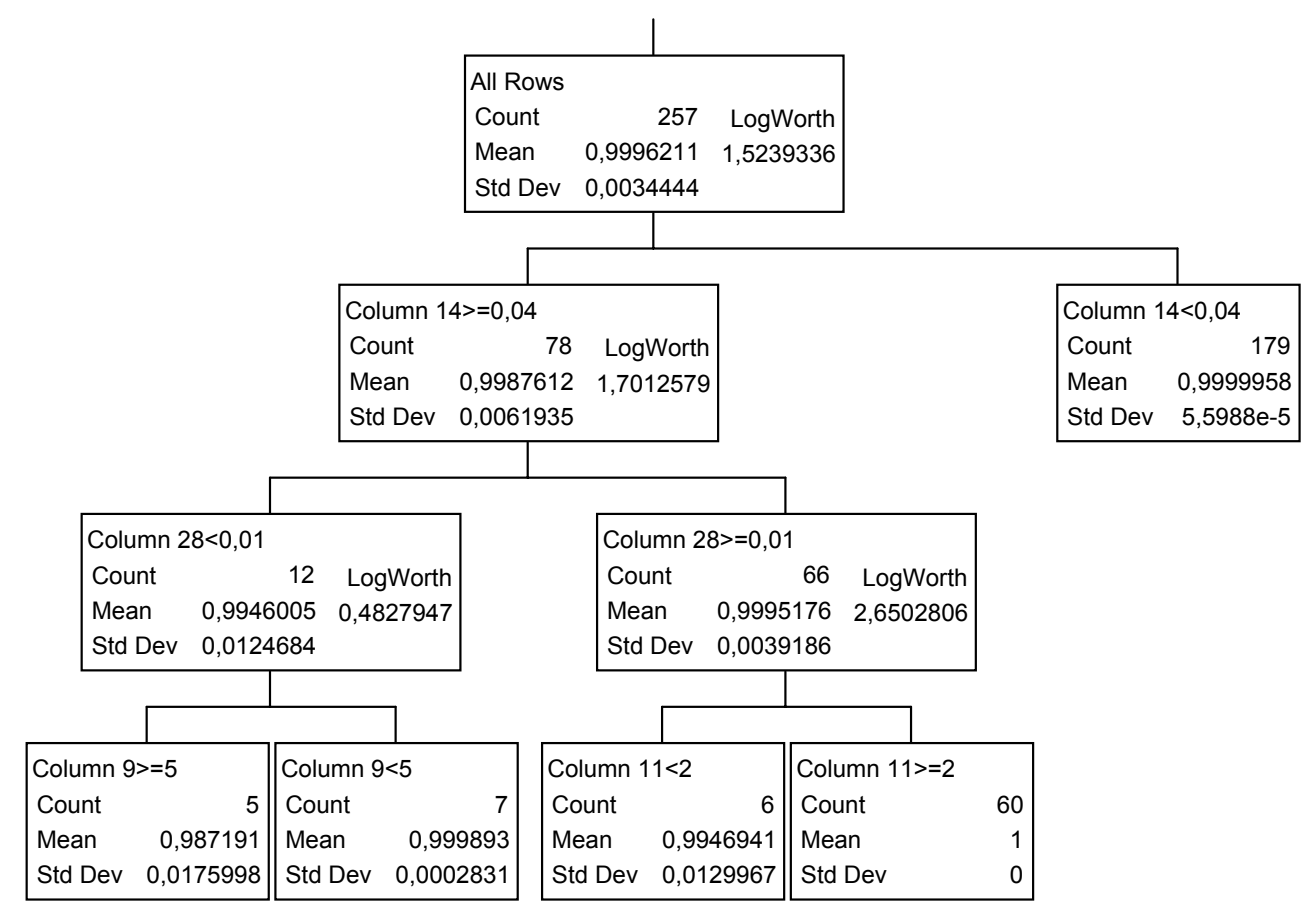

Figure 1: Regression Tree for Units B and C 


\section{FINAL REMARKS}

We have briefly described our simulation model for military deployment and showed the application of an intelligent design of experiments approach in the analysis of a typical deployment scenario. Not all the explanatory tools available in the further analysis of the deployment scenario could be elaborated upon here. Yet, we have presented the general approach of analysis using a NOLH design.

\section{ACKNOWLEDGMENTS}

The authors would like to thank Mr. Erhan Çınar and Mr. Murat Durmaz of BilgiGIS Ltd. of Ankara, Turkey for sharing their expertise in GIS, providing guidance and help in integrating their GIS software, GeoKIT, with our simulation model. In addition, we thank Prof. Thomas Lucas and COL. Andy Hernandez of NPS for their insights and help on NOLH designs.

\section{REFERENCES}

Akgün, İ. and Tansel, B. 2007. Optimization of Transportation Requirements in the Deployment of Military Units. Computers and Operations Research, Volume 34(4): $1158-1176$.

Buss, A. 2001. Basic Event Graph Modeling. Simulation News Europe, 31: 1-6.

Buss, A. and Sanchez, P. J. 2002. Building Complex Models With LEGOS (Listener Event Graph Objects). Proceedings of the 2002 Winter Simulation Conference, ed., E. Yücesan, C-H. Chen, J. L. Snowdon, and J. M. Charnes, 732-737. Institute of Electrical and Electronics Engineers, Piscataway, New Jersey.

Cioppa, T. M., and Lucas, T. W. 2007. Efficient Nearly Orthogonal and Space Filling Latin Hypercubes. Technometrics 49(1):.

Kang, K., Doerr, K. H., and Sanchez, S. M. 2006. A Design of Experiments Approach to Readiness Risk Analysis. Proceedings of the 2006 Winter Simulation Conference, ed., L. F. Perrone, F. P. Wieland, J. Liu, B. G. Lawson, D. M. Nicol, and R. M. Fujimoto, 1332-1339.Institute of Electrical and Electronics Engineers, Piscataway, New Jersey.

Kleijnen, J. P. C., Sanchez, S. M., Lucas, T. W., and Cioppa, T. M. 2005. A User's Guide to the Brave New World of Simulation Experiments. INFORMS Journal on Computing, 17(3): 263-289.

Sanchez, S. M. and Hernandez, A. S. 2005. NOLHdesigns Spreadsheet. Available online via <http://diana.cs.nps.navy.mil/SeedLa $\mathrm{b} />$ [accessed 12/19/2007].

SAS. 2005. JMP User's Guide, Version 6: SAS Institute Inc. Cary, NC, USA.
Sargent, R. G. 1988. Event Graph Modeling for Simulation with an Application to Flexible Manufacturing Systems. Management Science 34(10) : 1231-1251.

Sargent, R. G. 2001. Some Approaches and Paradigms for Verifying and Validating Simulation Models. Proceedings of the 2001 Winter Simulation Conference, ed., B. A. Peters, J.S. Smith, D. J. Mederios, and M. W. Rohrer, 106-114. Institute of Electrical and Electronics Engineers, Piscataway, New Jersey.

Schruben, L. 1983. Simulation Modeling with Event Graphs. Communications of the ACM 26(11): 957963.

Ye, K.Q. 1998. Orthogonal Column Latin Hypercubes and Their Applications in Computer Experiments. Journal of the American Statistical Association, 93: 14301439.

Yıldırım, U. Z, Sabuncuoğlu, İ., and Tansel, B. 2007. A Simulation Model for Military Deployment. Proceedings of the 2007 Winter Simulation Conference, eds., S.G. Henderson, B. Biller, M.-H. Hsieh, J. Shortle, J.D. Tew, and R.R. Barton, 1361-1369. Institute of Electrical and Electronics Engineers, Piscataway, New Jersey.

\section{AUTHOR BIOGRAPHIES}

UĞUR ZIYA YILDIRIM is a Major in the Turkish Army. He received his B.S. and M.S. in Operations Research from the U.S. Military Academy in 1991 and from the Naval Postgraduate School in 1999 respectively. He is currently a Ph.D. candidate at the Department of Industrial Engineering at Bilkent University in Ankara, Turkey. His e-mail address is <uziya@bilkent.edu.tr $>$.

İHSAN SABUNCUOĞLU received his Ph.D. degree in Industrial Engineering from the Wichita State University. Prof. Sabuncuoğlu teaches and conducts research in the areas of simulation, scheduling, AI, and manufacturing systems. He has been a visiting professor at the Wichita State. He has published papers in IIE Transactions, Decision Sciences, International Journal of Production Research, Simulation, Journal of Manufacturing Systems, International Journal of Flexible Manufacturing Systems, International Journal of Computer Integrated Manufacturing, Computers and Operations Research, European Journal of Operational Research, Journal of Operational Research Society, Computers and Industrial Engineering, International Journal of Production Economics, Journal of Intelligent Manufacturing, OMEGA-International Journal of Management Sciences, and Production Planning and Control. Honors Societies; Alpha Pi Mu (National Industrial Engineering Honor Society). Prof. Sabuncuoğlu is the chairman of the Industrial Engineering Department. His email address is <sabun@bilkent.edu.tr $>$. 
BARBAROS TANSEL received his Ph.D. degree in Industrial and Systems Engineering from the University of Florida, Gainesville (U.S.A.) in 1979. Prior to his appointment at Bilkent, he has served as a faculty member at the Middle East Technical University, Georgia Institute of Technology, and the University of Southern California. He has been a faculty member of the Department of Industrial Engineering at Bilkent University since 1991. His primary research interests are in network location theory, location and layout optimization, theory of optimization with imprecise data, hub location modeling and optimization, and logistics system design. Prof. Tansel's research articles have been published in Management Science, Operations Research, Transportation Science, IIE Transactions, Journal of the Operational Research Society, European Journal of Operational Research, International Journal of Production Research, and Journal of Manufacturing Systems. His e-mail address is <barbaros@bilkent.edu.tr>.

AHMET BALCIOĞLU is a Major in the Turkish Army. He received his B.S. in Business Administration from the Turkish Military Academy in 1993 and his M.S. in Operations Research from the Naval Postgraduate School in 2000. His e-mail address is<abalcioglu@kkk.tsk.mil.tr>. 